\title{
Advanced glycation end products-induced mitochondrial energy metabolism dysfunction alters proliferation of human umbilical vein endothelial cells
}

\author{
YUAN LI, YE CHANG, NING YE, YINTAO CHEN, NAIJIN ZHANG and YINGXIAN SUN
}

Department of Cardiology, The First Hospital of China Medical University, Heping, Shenyang, Liaoning 110001, P.R. China

Received February 3, 2016; Accepted January 20, 2017

DOI: $10.3892 / \mathrm{mmr} .2017 .6314$

\begin{abstract}
Advanced glycation end products (AGEs) restrain the proliferation of endothelial cells, which is an important determinant of diabetic vasculopathy. Mitochondrial biogenesis serves an essential role in cellular adaptation and repair. The current study aimed to investigate alterations in mitochondrial energy metabolism in human umbilical vein endothelial cells (HUVECs) and the latent mechanism regulated by AGEs. The proliferation of cultured HUVECs stimulated with AGEs was detected using an MTT assay and a real-time cell analyzer (RTCA). Mitochondrial energy metabolism was measured using a Seahorse metabolic flux analyzer. Mitochondrial membrane potential was detected under fluorescence microscopy following staining with tetraethylrhodamine and MitoTracker Red. Respiratory chain complexes I-V were detected using western blotting. MTT and RTCA assays demonstrated that AGEs treatment significantly inhibited the viability and proliferation of HUVECs when compared with bovine serum albumin treatment. Results from the Seahorse metabolic flux analyzer indicated that mitochondrial aerobic respiration and glycolysis declined following AGEs treatment. In addition, mitochondrial membrane potential and the expression of mitochondrial respiration chain complexes I/II/III/IV/V notably decreased in the presence of AGEs. In conclusion, the results of the present study indicated that AGEs exhibited an inhibitory effect on the proliferation in HUVECs potentially by mediating the dysfunction of mitochondrial energy metabolism and glycolysis. This may provide a new consideration for therapeutic methods in diabetic vascular complications.
\end{abstract}

Correspondence to: Professor Yingxian Sun, Department of Cardiology, The First Hospital of China Medical University, 155 Nanjing North Street, Heping, Shenyang, Liaoning 110001, P.R. China

E-mail: sunyingxian12@126.com

Key words: advanced glycation end products, diabetes mellitus, mitochondrial energy metabolism, human umbilical vein endothelial cells, glycolysis

\section{Introduction}

Diabetes has become one of the three major chronic diseases, along with hypertension and coronary heart disease, that seriously endanger human health (1). Diabetic complications, comprising macroangiopathy and microangiopathy, involve multiple organs, including the heart, brain, kidney and eyes, and are the primary underlying causes of increased mortality and disability rates in patients with diabetes. The basic pathological process of diabetes is atherosclerosis (2). It has been reported $(3,4)$ that the level of advanced glycation end products (AGEs) in vivo was clearly associated with the severity of diabetic complications and atherosclerosis.

AGEs are formed following long-term exposure to high levels of sugar by a series of reactions and structural rearrangements of non-enzymatic glycosylation and lipid oxidation. While the glycosylation reaction occurs slowly in normal organisms, the level of AGEs gradually increases with age $(5,6)$. However, during the aging process, particularly in the case of long-term high blood glucose, the glycosylation reaction and subsequent formation of AGEs increases. Thus, it is acknowledged that this reaction is an inducing factor of diabetic complications (7). In physiological conditions, AGEs are degraded to soluble polypeptides by the mononuclear macrophage system via endocytosis or extracellular protein degradation systems, and are primarily cleared by the kidneys (8). However, in pathological conditions, particularly diabetic complications, AGEs yet to be eliminated may stimulate the secretion of cytokines from macrophage and mesangial cells, thus leading to vascular hyperplasia, mesangial proliferation and glomerular hypertrophy, which are associated with the development of diabetic nephropathy (9). In addition, AGEs may modify numerous additional proteins, leading to a number of diseases. Glycosylated high density lipoprotein cholesterol decreases the ability to reverse cholesterol transport, as well as increasing the oxidation of low density lipoprotein cholesterol by reducing the activity of paraoxonase, which promotes the development of vessel wall damage $(10,11)$. In addition, AGEs combine with receptors for advanced glycation end products (RAGE), which leads to the activation of multiple intracellular signaling pathways and serves a key role in the pathogenesis of several diseases, including atherosclerosis (8), diabetes complications (12), osteoporosis (13) and cancer $(14,15)$. 
In diabetes complications or atherosclerosis, increasing evidence suggests that endothelial dysfunction may be a key factor. Endothelial dysfunction leads to inflammation, oxidative stress and cell death, which result in vascular remodeling and subsequent vascular diseases. It has been reported (16-18) that AGEs bind to RAGE to induce oxidative stress, inflammation and apoptosis that lead to endothelium damage. Therefore, exploring the potential mechanism of AGEs-induced endothelial dysfunction is clearly significant for the prevention and treatment of diabetic vascular damage.

Mitochondria exist in the majority of cells and possess their own genome, termed mitochondrial DNA (mtDNA), and a double-membrane organization that is required to produce energy for conducting cellular aerobic respiration. In mammals, mtDNA encodes 13 key structural subunits required for the catalytic activity of four out of five oxidative phosphorylation (OXPHOS) enzyme complexes I, III, IV and V (19). The OXPHOS enzyme complexes serve a critical role in electron transfer in the respiratory chain (20). In addition to providing adenosine 5'-triphosphate (ATP), mitochondria may serve as an important source of reactive oxygen species (ROS) and regulate various cellular process, including cell proliferation (21), cell signal transduction and apoptotic cell death (22). Mitochondrial-derived ROS (mROS) appear to serve a cell signaling function when maintained at low levels. However, under pathological stresses, the production of excessive mROS may contribute to decreased mitochondrial quality via several interrelated mechanisms, including mitochondrial calcium overload, increased levels of ROS, endoplasmic reticulum stress and the accumulation of aggregated proteins. That oxidative damage impairs the quality of the mitochondrial population has been demonstrated by the increased harm to mtDNA, decreased membrane potential and a diminished bioenergetic reserve capacity that may be conducive to damage of the complexes $(20,23-25)$. Nevertheless, cells take immediate measures in response to the accumulation of impaired mitochondria. According to previous studies $(26,27)$, two main pathways are involved in this process. First, intramitochondrial proteases enable degradation of the oxidized proteins, including ATP-stimulated mitochondrial Lon protease (27). The mitochondrial ATPases associated with various cellular activities have been reported to serve a vital role in preventing mtDNA escaping from mitochondria to the nucleus ensuring appropriate fission and fusion, in addition to expeditious proteolysis of non-assembled inner membrane proteins (27). Second, in response to more extensive mitochondrial damage, elevated mitochondrial fragmentation and decreased fusion may separate damaged mitochondrial proteins, lipids and DNA from functional components, and the entire damaged organelle is processed by mitophagy (26). Previously, Kizhakekuttu et al (28) reported that mitochondrial dysfunction serves a central role in endothelial dysfunction in diabetes mellitus, which was evidenced by lower mitochondrial $\mathrm{O}_{2}$ consumption, mitochondrial membrane potential, glutathione/glutathione disulfide ratio and higher mROS production in an additional study (29). Furthermore, 4-hydroxynonenal, one of the oxidized lipids, contributes to mytocyte injury by decreasing the bioenergetic reserve capacity (20). Together these results indicate that mitochondrial energetic metabolism may serve an essential role in cellular dysfunction. Therefore, the purpose of the present study was to explore whether AGEs may induce the dysfunction of human umbilical vein endothelial cells (HUVECs) through mitochondrial dysfunction and to determine the effect of AGEs on mitochondrial aerobic respiration and glycolysis.

In summary, the primary aim was to explore the effect of AGEs on the proliferation of HUVECs and to assess whether mitochondrial metabolism may serve a key role in this process, as well as the potential underlying mechanisms involved.

\section{Materials and methods}

Materials. A Seahorse metabolic flux analyzer (Seahorse Bioscience; Agilent Technologies, Inc., Santa Clara, CA, USA), LSM510 confocal microscope (Zeiss AG, Thornwood, NY, USA), MicroChemi 4.2 (DNR Bio-Imaging Systems, Ltd., Jerusalem, Israel) and bovine serum albumin (BSA) and AGEs-BSA (Calbiochem; Merck KGaA, Darmstadt, Germany) were used in the present study and obtained from their respective manufacturers. All materials and reagents for the extracellular flux assays were purchased from Seahorse Bioscience (Agilent Technologies, Inc.). All antibodies, staining and additional reagents used in the present study were obtained from Sigma-Aldrich (Merck KGaA) unless otherwise specified.

Cell culture and treatment. Primary HUVECs (S200-05N; Sigma-Aldrich; Merck KGaA) were cultured at $37^{\circ} \mathrm{C}$ in a humidified atmosphere and $5 \% \mathrm{CO}_{2}$ in Dulbecco's modified Eagle's medium (DMEM; HyClone; GE Healthcare Life Sciences, Logan, UT, USA) containing $10 \%$ fetal bovine serum (FBS; HyClone; GE Healthcare Life Sciences). HUVECs were incubated for 3 days until 70-80\% confluence, before they were starved in serum-free Basal Medium Eagle (B9638; Sigma-Aldrich; Merck KGaA) for $24 \mathrm{~h}$. Cells treated with BSA served as a control. Cells in the AGEs-treated group were incubated with AGEs-BSA $(100 \mu \mathrm{g} / \mathrm{ml})$ for varying durations $(12,24$ and $48 \mathrm{~h})$, while cells in the control group were treated with BSA $(100 \mu \mathrm{g} / \mathrm{ml})$ for $24 \mathrm{~h}$.

Cell viability assays. For cell viability assays, cells were plated in 96 -well dishes $\left(1 \times 10^{4}\right.$ cells/well) and incubated overnight. The following day, cells were treated with AGEs in media containing $10 \%$ FBS and incubated for a further 12, 24 and 48 h. Cell viability was assessed using the MTT assay (Chemicon; EMD Millipore, Billerica, MA, USA) according to the manufacturer's protocol. The absorbance was measured with a microplate reader (Bio-Rad, Hercules, CA, USA) at $570 \mathrm{~nm}$.

Growth curve assays using the real-time cell analyzer (RTCA). Growth curve assays were performed in real-time and in quadruplicate using the xCELLigence system (ACEA Biosciences, Inc., San Diego, CA, USA). RTCA E-plates (ACEA Biosciences, Inc.) were seeded with 5,000 cells/well. After all the chambers were set up, the RTCA E-plates were put into the xCELLigence instrument in an incubator at $37^{\circ} \mathrm{C}$ and $5 \% \mathrm{CO}_{2}$. Cell growth was reported as the cell index, which was a dimensionless, relative measure of impedance reflecting the number of viable adherent cells, with a consistent logarithmic 
association with cell number. Cell index was recorded automatically every $15 \mathrm{~min}$ and monitored continuously for $48 \mathrm{~h}$.

XF Cell Mito Stress test using the XF24 Extracellular Flux analyzer. An XF24 Analyzer (Seahorse Bioscience; Agilent Technologies, Inc.) was used to measure bioenergetic function in HUVECs. The XF24 possesses a transient $2 \mu 1$ chamber in specialized microplates that allows for the measurement of the oxygen consumption rate (OCR) and extracellular acidification rate (ECAR) or proton production rate in real-time (30). The cells were seeded at a density of 20,000 cells/well. Following adherence for $4 \mathrm{~h}$, AGEs or BSA was added to the microplates for co-incubation with cells for $24 \mathrm{~h}$, and $1 \mu \mathrm{M}$ oligomycin, $0.5 \mu \mathrm{M}$ carbonyl cyanide-4-(trifluoromethoxy) phenylhydrazone (FCCP) and $0.5 \mu \mathrm{M}$ rotenone/antimycin A were subsequently added. The sensor cartridge was hydrated in XF Calibrant at $37^{\circ} \mathrm{C}$ in a non- $\mathrm{CO}_{2}$ incubator overnight. The culture medium was refreshed at $1 \mathrm{~h}$ prior to all bioenergetic assays using unbuffered DMEM (pH 7.4) supplemented with 4 mM L-glutamine (Seahorse Bioscience; Agilent Technologies, Inc.).

To test mitochondrial respiration, a XF Cell Mito Stress Test kit (Seahorse Bioscience; Agilent Technologies, Inc.) was used according to the manufacturer's protocol. Briefly, $1 \mu \mathrm{M}$ oligomycin (final concentration), $0.5 \mu \mathrm{M}$ FCCP (final concentration) and $0.5 \mu \mathrm{M}$ rotenone and antimycin A (final concentration) were subsequently added to the microplates. This enabled determination of the basal level of oxygen consumption, ATP-linked oxygen consumption, non-ATP-linked oxygen consumption, the maximal respiration capacity and the non-mitochondrial oxygen consumption. A total of three basal OCR measurements were recorded prior to the injection of oligomycin. The decreased level of OCR represented oligomycin-sensitive OCR due to its inhibition of ATP synthase (complex V). FCCP, an uncoupling protein, was then injected and the FCCP-stimulated OCR was used to calculate spare respiratory capacity, which was defined as the difference between maximal respiration and basal respiration. The third injection was a mixture of rotenone (a complex I inhibitor) and antimycin A (a complex III inhibitor). This combination inhibited mitochondrial respiration completely, and thus no oxygen was further consumed by cytochrome $c$ oxidase. The remaining OCR measurement obtained following this treatment was primarily non-mitochondrial and may have been due to cytosolic oxidase enzymes.

XF Glycolysis Stress Test using the XF24 Extracellular Flux Analyzer. The XF Glycolysis Stress Test (Seahorse Bioscience; Agilent Technologies, Inc.) was used to assess glycolysis function in cells, which was conducted using the XF24 Analyzer. By directly measuring ECAR, the kit provided a standard method to assess the following key parameters of glycolysis flux: Glycolysis, glycolytic capacity and glycolytic reserve, in addition to non-glycolytic acidification. Cells were seeded at a density of 20,000 cells/well. Cells were first incubated in pyruvate-free glycolytic assay medium for $1 \mathrm{~h}$ prior to the first injection of a saturated concentration of glucose (final concentration: $10 \mathrm{mM}$ ). The cells catabolize glucose into pyruvate via the glycolysis pathway, producing ATP, nicotinamide-adenine dinucleotide (reduced form), water and protons. The discharge of protons into surrounding medium leads to a sudden increase in ECAR, which was used to define the basal glycolytic capacity. The second injection was oligomycin (final concentration: $1 \mu \mathrm{M}$ ), which may divert energy production to glycolysis by restricting mitochondrial ATP production. Consequently, the sharp increase in ECAR indicates the level of glycolytic capacity. The final injection was 2-deoxy-glucose (2-DG; final concentration: $50 \mathrm{mM}$ ), which is a glucose analog that inhibits glycolysis through competitive binding to glucose hexokinase; the first enzyme in the glycolytic pathway. The resulting decrease in ECAR confirmed that the ECAR produced in the experiment was caused by glycolysis. The gap between glycolytic capacity and glycolysis was defined as the glycolytic reserve. The ECAR prior to glucose injection is referred to as non-glycolytic acidification, and may occur due to additional processes in the cell.

Measurement of mitochondrial membrane potential. Tetraethylrhodamine (TMRE) and MitoTracker Red were used to detect changes in mitochondrial membrane potential, as previously described $(31,32)$. Briefly, cells were seeded into a 6 -wells plate at a density of 5,000/well. Then cells were cultured in the presence of either $100 \mu \mathrm{g} / \mathrm{ml} \mathrm{BSA}$ or $100 \mu \mathrm{g} / \mathrm{ml}$ AGEs for $24 \mathrm{~h}$. The old culture media was removed and cells were incubated with $100 \mathrm{nM}$ TMRE or $200 \mathrm{nM}$ MitoTracker Red for $30 \mathrm{~min}$ at $37^{\circ} \mathrm{C}$ and $5 \% \mathrm{CO}_{2}$. In the last $5 \mathrm{~min}$, cells were incubated with Hoechst 33,342 (final concentration: $8 \mu \mathrm{g} / \mathrm{ml}$ ) for $5 \mathrm{~min}$ at $37^{\circ} \mathrm{C}$ and $5 \% \mathrm{CO}_{2}$ to stain the nuclei. Following washing with PBS, HUVECs were analyzed under a fluorescence microscope.

Western blot analysis. Cells were lysed in lysis buffer $(20 \mathrm{mM}$ Tris-HCl, $150 \mathrm{mM} \mathrm{NaCl}, 2$ mM EDTA and 1\% Triton X-100) containing a protease inhibitor cocktail (Sigma-Aldrich; Merck KGaA). Cell protein extracts were quantified using a bicinchoninic acid protein assay kit (Sigma-Aldrich; Merck $\mathrm{KGaA})$. Equal quantities of protein $(20 \mu \mathrm{g})$ were separated by $12 \%$ SDS-PAGE and transferred to polyvinylidene difluoride (PVDF) membranes (EMD Millipore, Billerica, MA, USA). Then the PVDF membranes were blocked with 5\% nonfat milk in Tris-buffered solution (TBS) for $1.5 \mathrm{~h}$ at the room temperature. After washing with $1 \mathrm{X}$ TBS (three times, $15 \mathrm{~min} /$ time), the membranes were incubated with primary antibodies against OXPHOS (ab110413; 1:1,000; Abcam, Cambridge, UK) or GAPDH (AB2302; 1:1,000; EMD Millipore, Billerica, MA, USA) at $4{ }^{\circ} \mathrm{C}$ overnight. After washing with $1 \mathrm{X}$ TBS (three times, $15 \mathrm{~min} /$ time), the membranes were incubated with horseradish peroxidase-conjugated anti-mouse or anti-rabbit secondary antibodies (AP181R for anti-mouse and AP187R for anti-rabbit; 1:10,000; EMD Millipore) for $1.5 \mathrm{~h}$ at room temperature. Finally, enhanced chemiluminescence solutions (GE Healthcare Life Sciences, Chalfont, UK) was used to detect immunoreactive binding. The band intensity was quantified with Image J software version 1.47 (NIH, Bethesda, MD, USA).

Statistical analysis. All data were obtained from $>3$ independent experiments. Comparisons between two groups were analyzed using the unpaired Student's t-test. Comparisons among multiple groups were analyzed using one-way analysis 
of variance with Bonferroni post hoc tests where applicable. All the statistical analyses were performed using SPSS 17.0 (SPSS, Inc., Chicago, IL, USA). P<0.05 was considered to indicate a statistically significant difference.

\section{Results}

AGEs inhibited the proliferation of HUVECs. In order to determine the effect of AGEs on the growth of HUVECs, HUVECs were treated with AGEs $(100 \mu \mathrm{g} / \mathrm{ml})$ or BSA $(100 \mu \mathrm{g} / \mathrm{ml})$ for $24 \mathrm{~h}$ and cell proliferation was assessed using MTT and RTCA assays. MTT assay analysis demonstrated that the viability of HUVECs decreased in a time-dependent manner following AGEs treatment (Fig. 1A). Similarly, the RTCA proliferation assay demonstrated that the cell index was significantly lower in AGEs $(100 \mu \mathrm{g} / \mathrm{ml})$-group when compared with BSA-group following treatment for $48 \mathrm{~h}$ (Fig. 1B). These results suggest that AGEs reduces the proliferation of HUVECs in a time-dependent manner.

Effect of AGEs on mitochondrial aerobic metabolism. In order to investigate the specific negative effects of AGEs on mitochondrial aerobic metabolism in HUVECs, the XF Cell Mito Stress assay was employed. HUVECs were treated with AGEs for $24 \mathrm{~h}$ prior to exposure to $1 \mu \mathrm{M}$ oligomycin, $0.5 \mu \mathrm{M}$ FCCP and $0.5 \mu \mathrm{M}$ rotenone and antimycin A at various time points. As demonstrated in Fig. 2, AGEs reduced the OCR prior to the injection of oligomycin, suggesting that AGEs prohibited mitochondrial aerobic respiration. In addition, HUVECs treated with AGEs exhibited an increase in basal oxygen consumption and proton leakage when compared with the BSA-treated cells (Fig. 2C). By contrast, a significant reduction in maximal respiration capacity, spare respiration capacity and non-mitochondrial respiration was observed in the AGEs-treated cells when compared with the BSA-treated cells (Fig. 2C).

Effect of AGEs on glycolytic function. In addition to the OCR, the XF Cell Mito Stress assay facilitated the measurement of protons that were produced by HUVEC cells, which is an indicator of lactate production and may therefore be used as an index of glycolysis (30). HUVECs were treated with AGEs for $24 \mathrm{~h}$ prior to exposure to $10 \mathrm{mM}$ glucose, $1 \mu \mathrm{M}$ oligomycin and $50 \mathrm{mM}$ 2-DG at various time points, as demonstrated in Fig. 3A. AGEs demonstrated an inhibitory effect on glycolytic function (Fig. 3B). The key parameters of glycolysis, glycolytic capacity, glycolytic reserve and non-glycolytic acidification were decreased in the AGEs group compared with the BSA-treated control group, indicating that AGEs inhibited glycolysis (Fig. 3C).

Inhibitory effect of AGEs on OCR and ECAR in HUVECs. In order to assess the influence of AGEs on mitochondrial bioenergetic metabolism and anaerobic glycolysis, extracellular flux analysis was used to determine rates of $\mathrm{O}_{2}$ consumption. Cells were incubated with AGEs $(100 \mu \mathrm{g} / \mathrm{ml})$ or BSA $(100 \mu \mathrm{g} / \mathrm{ml})$ in the specialized microplates, and the XF24 assay was performed to measure real-time OCR and ECAR over $24 \mathrm{~h}$. As demonstrated in Fig. 4A, the OCR decreased in response to AGEs when compared with BSA over the course of the analysis. Unexpectedly, ECAR in the AGEs-treated group was lower compared with the BSA-treated cells (Fig. 4B), which suggests that significant cellular damage or even cell death may occur in HUVECs exposed to AGEs.

Effect of AGEs on the mitochondrial membrane potential and mitochondrial respiration chain complex. Since AGEs exhibited an inhibitory effect on aerobic metabolism, the next aim of the present study was to investigate whether AGEs affected mitochondrial function. In order to determine whether AGEs affected the mitochondrial membrane potential, cells were labeled with TMRE or MitoTracker Red and HUVEC nuclei were counterstained with Hoechst stain. TMRE and MitoTracker Red staining demonstrated that the mitochondrial membrane potential declined following treatment with AGEs (Fig. 5A and B). In order to investigate the influence of AGEs on mitochondrial respiration chain complexes, western blotting was performed to detect the protein expression levels of complexes I-V. Fig. 5C and D demonstrates that the expression levels of complexes I-V were significantly reduced in the AGEs group when compared with the BSA group (Fig. 5C and D).

\section{Discussion}

The present study utilized a newly-emerging assay for the determination of mitochondrial function in intact HUVECs. To the best of the authors' knowledge, the results of the current study demonstrate for the first time the precise energy metabolism and mitochondrial dysfunction elicited by AGEs in endothelial cells.

AGEs serve a detrimental role in the pathogenesis of diabetic complications and atherosclerosis $(5,33)$. The majority of studies $(6,34-36)$ have demonstrated that AGEs induce harmful effects during endothelial dysfunction, including inhibited proliferation, apoptosis, increased inflammatory response and endoplasmic reticulum and oxidative stress, via various signaling pathways. An increasing number of studies [reviewed in (37)] have focused on mitochondrial dysfunction induced by AGEs in endothelial cells. However, few studies have investigating the effect of AGEs on mitochondrial function and the precise alterations in energy metabolism in HUVECs have been conducted. In the present study, extracellular flux analysis, which is a recent mainstream method for measuring mitochondrial function in cells and tissues, was used to monitor the mitochondrial function of intact HUVECs. The results revealed that AGEs significantly inhibit the viability and proliferation of HUVECs potentially via an energy deficiency due to decreasing mitochondrial aerobic respiration and glycolysis.

Mitochondria are the metabolic powerhouses of the cell, providing ATP for inducing reactions and maintaining core metabolites for the fundamental survival of cells. Mitochondria regulate various cellular processes, including proliferation, apoptosis and ROS generation $(21,22)$. Under pathological conditions, several interacting mechanisms facilitate a decline in mitochondrial quality, as evidenced by the decreased mitochondrial potential, increased mtDNA damage and destruction of bioenergetic reserve capacity (38). Defects in mitochondrial biogenesis and dynamics serve a detrimental role in the bioenergy supply and appear to be a contributor to endothelial cell dysfunction and the pathogenesis of cardiovascular diseases $(39,40)$. In the present study, 
A

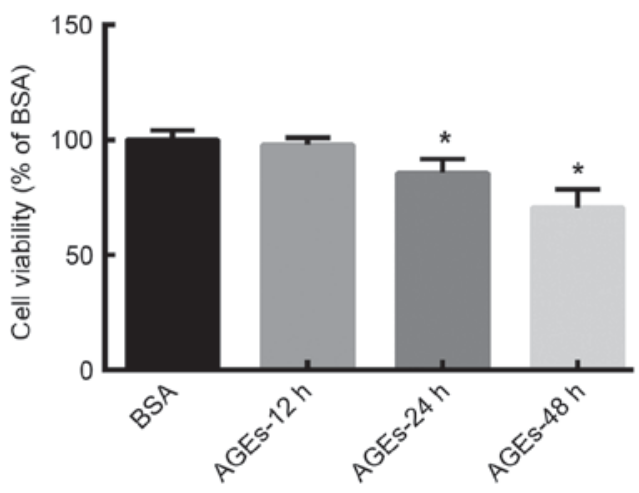

B

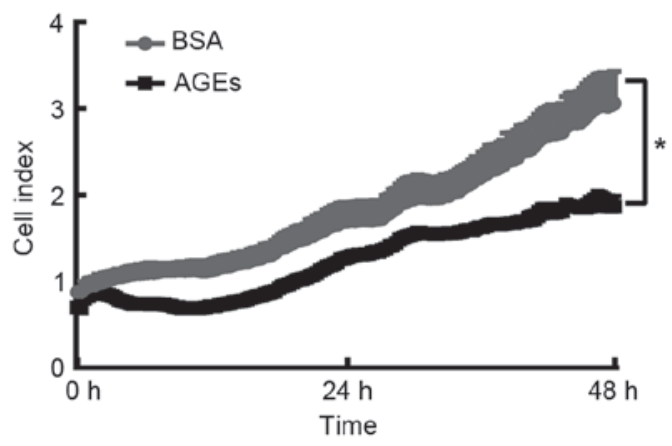

Figure 1. Antiproliferative activity of AGEs in HUVECs. Cells were cultured in the presence of AGEs (100 $\mu \mathrm{g} / \mathrm{ml})$ or BSA (100 $\mu \mathrm{g} / \mathrm{ml})$ and cell viability was assessed using the (A) MTT assay and the (B) RTCA cell index. Data are presented as the mean \pm standard deviation (n $>3$ ). "P<0.05 vs. BSA group. AGEs, advanced glycation end products; HUVECs, human umbilical vein endothelial cells; BSA, bovine serum albumin; RTCA, real-time cell analyzer.

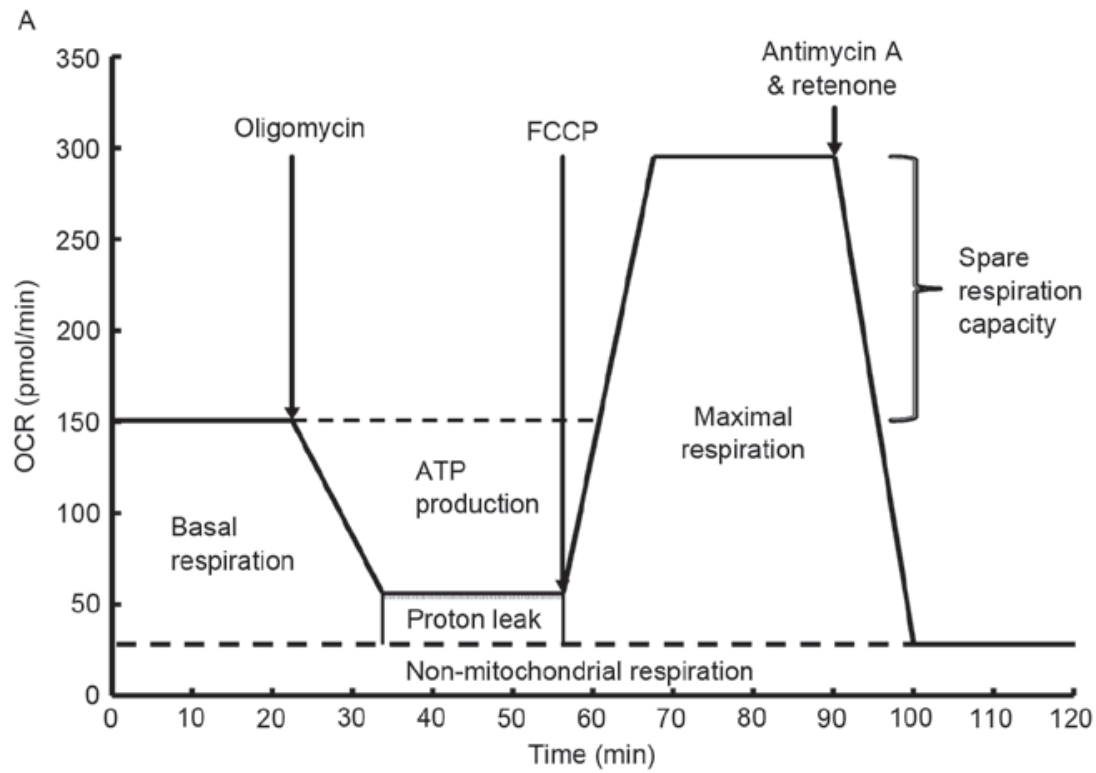

B

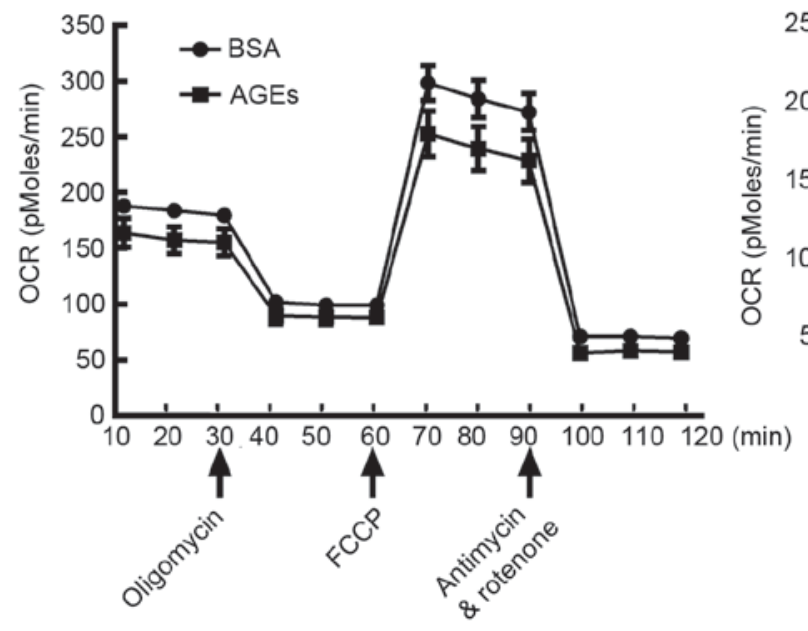

C

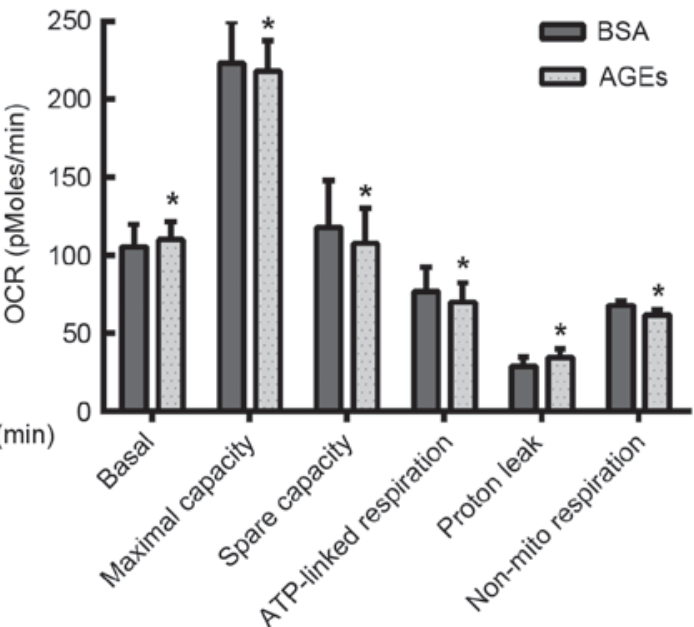

Figure 2. Measurement of mitochondrial aerobic respiration profile using the XF24 Extracellular Flux Analyzer. (A) Schematic of the XF Mito Stress Test used to determine the OCR. (B) HUVECs were seeded in the Seahorse Bioscience microplates (20,000 cells/well). Following adherence for $4 \mathrm{~h}$, AGEs or BSA was added to the microplates for co-incubation with cells for $24 \mathrm{~h}$, and $1 \mu \mathrm{M}$ oligomycin, $0.5 \mu \mathrm{M}$ FCCP and $0.5 \mu \mathrm{M}$ rotenone/antimycin A were subsequently added. (C) Individual parameters for respiration, including basal respiration, maximal respiration, spare respiration capacity, ATP-linked oxygen consumption, proton leakage and non-mito respiration in HUVECs. Each data point represents an OCR measurement. Data are presented as the mean \pm standard deviation $(\mathrm{n}=4)$. "P<0.05 vs. BSA group. OCR, oxygen consumption rate; HUVECs, human umbilical vein endothelial cells; AGEs, advanced glycation end products; BSA, bovine serum albumin; FCCP, carbonyl cyanide-4-(trifluoromethoxy) phenylhydrazone; ATP, adenosine 5'-triphosphate; Non-mito, non-mitochondrial. 
A

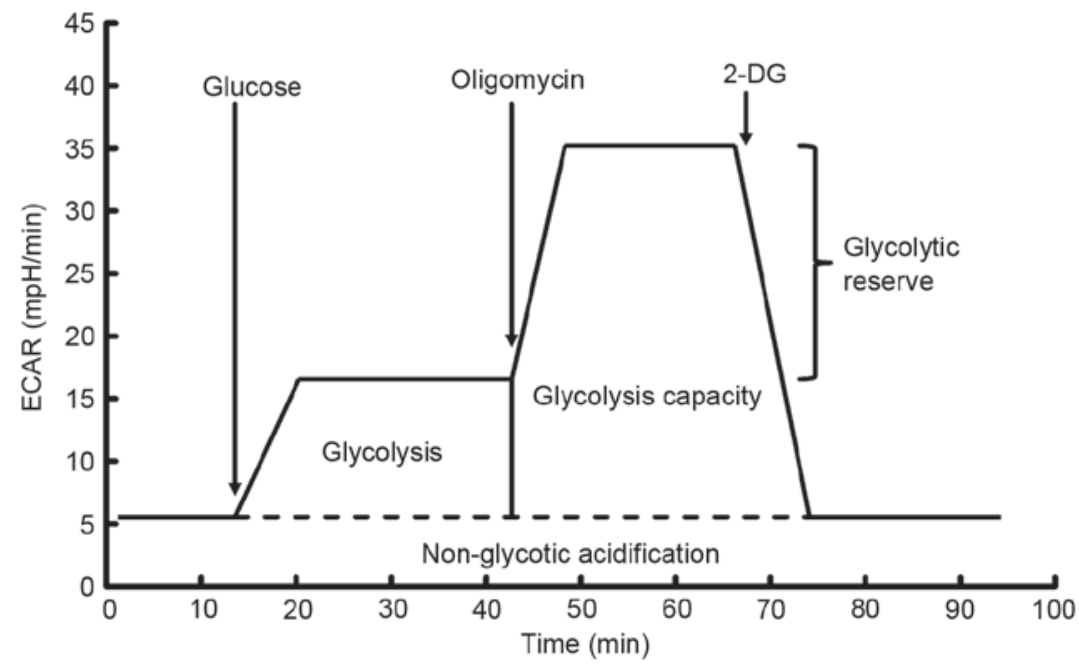

B

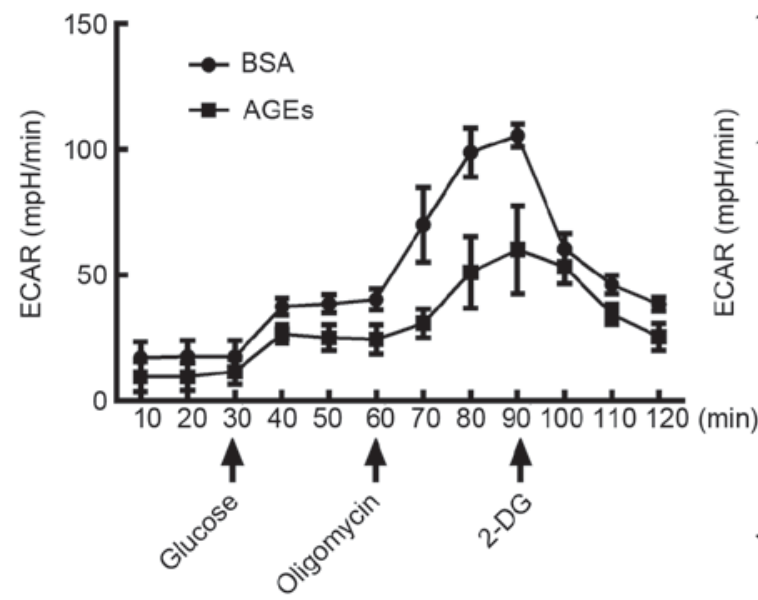

C

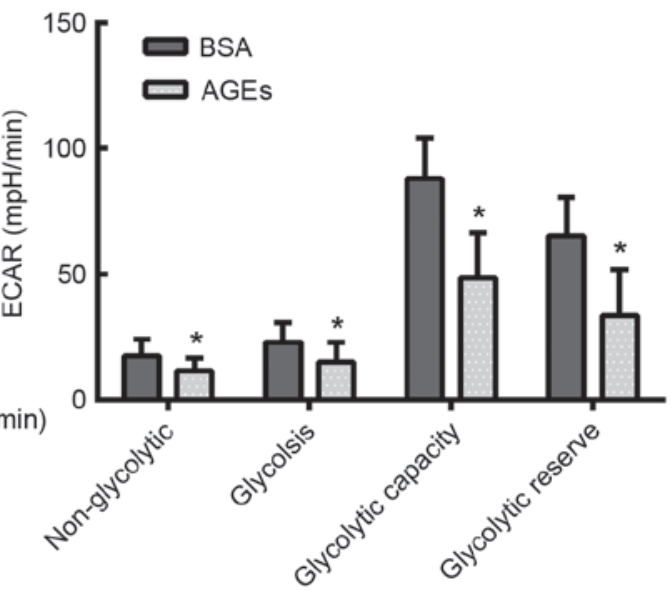

Figure 3. The profile of glycolysis in HUVECs. (A) Schematic of the mitochondrial stress test. (B) Alterations in the ECAR and (C) specific parameters for non-glycolytic acidification, glycolysis, glycolytic capacity and glycolytic reserve in HUVECs treated with AGEs or BSA. Cells were seeded at 20,000 cells/well and incubated with AGEs or BSA for $24 \mathrm{~h}$. ECAR was tested under the basal conditions with sequential addition of $15 \mathrm{mM}$ glucose, $2 \mu \mathrm{M}$ oligomycin and $50 \mathrm{mM}$ 2-DG. Each data point represents an ECAR value. Data are presented as the mean \pm standard deviation ( $\mathrm{n}=4$ ). ${ }^{*} \mathrm{P}<0.05$ vs. BSA group. HUVECs, human umbilical vein endothelial cells; AGEs, advanced glycation end products; BSA, bovine serum albumin; ECAR, extracellular acidification rate; 2-DG, 2-deoxy-glucose.
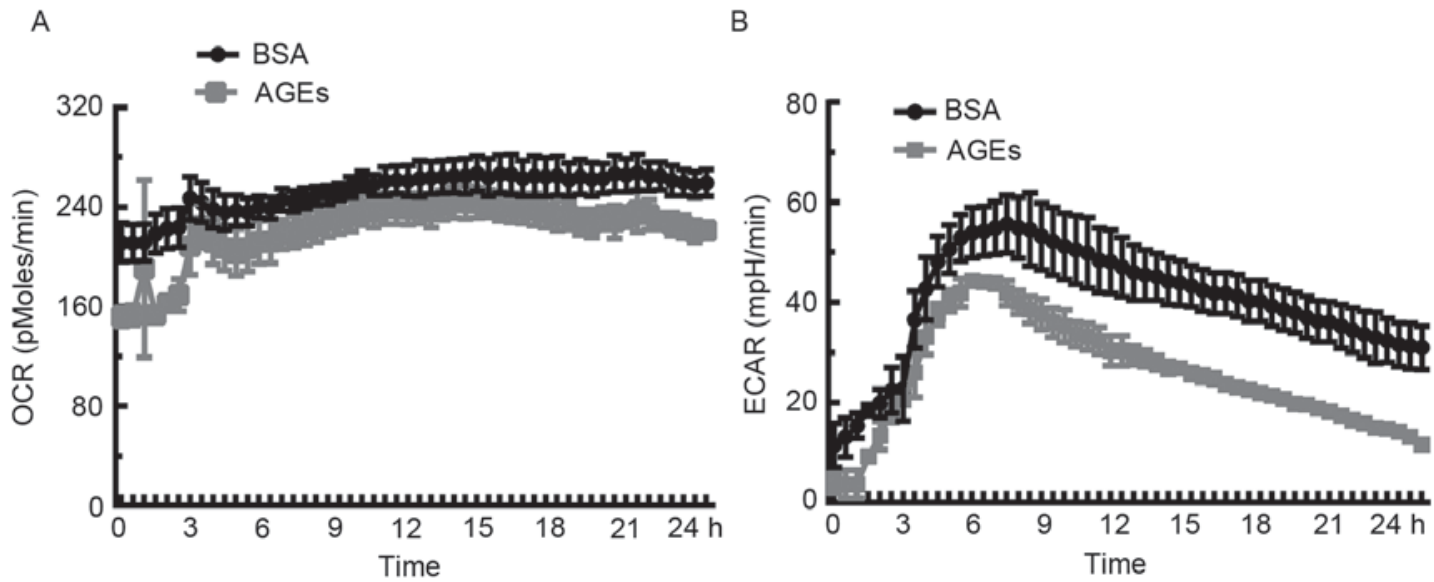

Figure 4. The effect of AGEs on the OCR and ECAR over $24 \mathrm{~h}$. HUVECs were seeded at a density of 20,000 cells/well and cultured for $4 \mathrm{~h}$. Real-time measurements of (A) OCR and (B) ECAR were obtained using the XF24 analyzer following the treatment with AGEs or BSA for $24 \mathrm{~h}$. Data are presented as the mean \pm standard deviation $(\mathrm{n} \geq 3)$. " $\mathrm{P}<0.05$ vs. BSA group. AGEs, advanced glycation end products; BSA, bovine serum albumin; HUVECs, human umbilical vein endothelial cells; OCR, oxygen consumption rate; ECAR, extracellular acidification rate. 

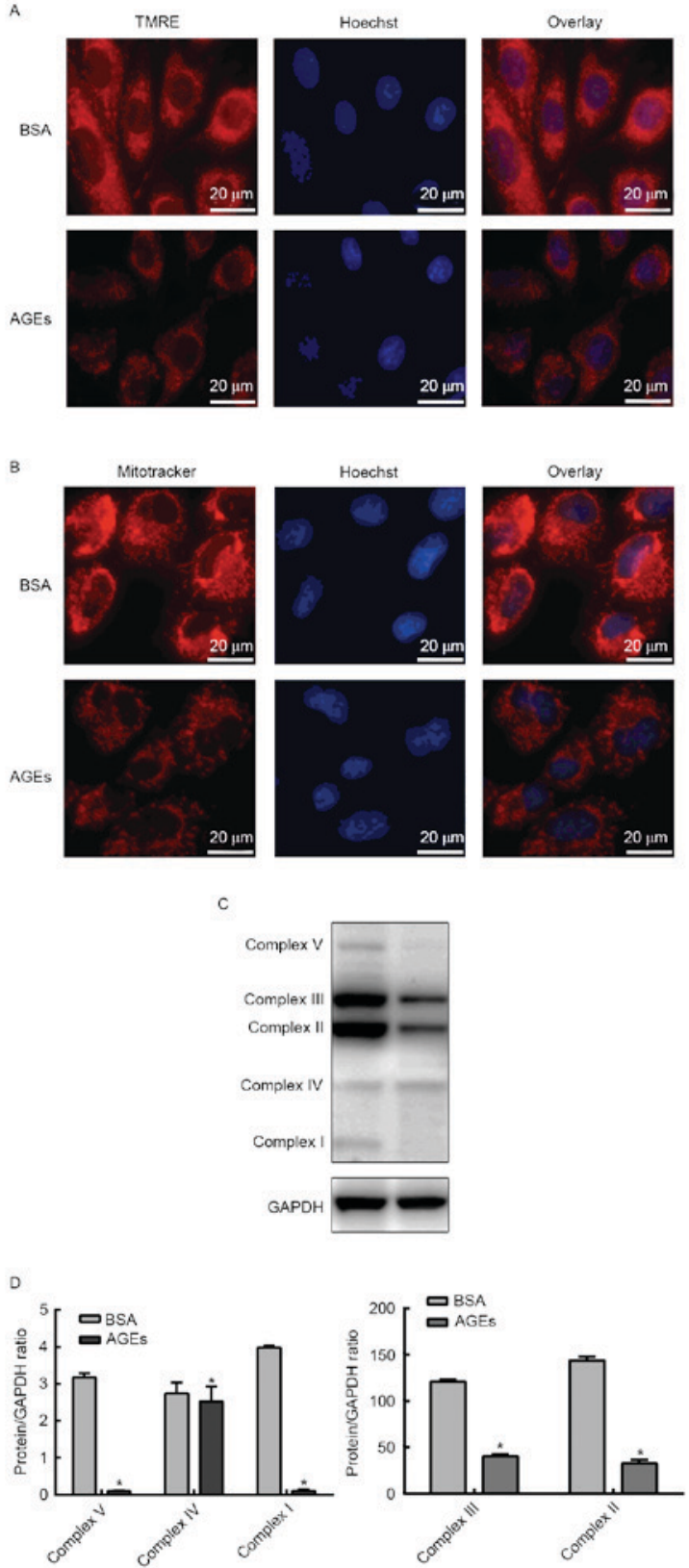

Figure 5. Effect of AGEs on mitochondrial membrane potential and mitochondrial respiratory chain complexes in HUVECs. (A) TMRE (red) and (B) MitoTracker Red staining of the mitochondria in HUVECs and counterstaining of nuclei with Hoechst stain (blue). (C) Western blot analysis of mitochondrial respiration chain complex I-V protein expression levels and (D) quantification of the results. The experiments were repeated $\geq 3$ times with reproducible results. Data are presented as the mean \pm standard deviation. ${ }^{*} \mathrm{P}<0.05$ vs. BSA control. AGEs, advanced glycation end products; HUVECs, human umbilical vein endothelial cells; TMRE, tetraethylrhodamine; BSA, bovine serum albumin.

the effect of AGEs on the mitochondrial aerobic metabolism and mitochondrial membrane potential profiles were analyzed in order to investigate the mechanism of AGEs-induced cell dysfunction associated with mitochondrial dysfunction. A previous review (41) confirmed that oxidative stress serves a fundamental role in AGEs-induced cell death. The results of the present study demonstrated that AGEs-induced oxidative stress elevated proton leakage thus resulting in a decline of mitochondrial membrane potential. In addition, the results demonstrated that AGEs may damage the OXPHOS system encoded by mtDNA. It is possible that the impaired mitochondrial respiration chain in AGEs-treated HUVECs was unable to transfer electrons to oxygen, which is the major factor in the production of ATP, resulting in the observed increase in basal respiration capacity, ATP-linked oxygen consumption and spare respiration capacity.

Of particular note, endothelial cells are known to possess a relatively low mitochondrial content and depend primarily on glycolysis (42). As energy deficiency occurs in cells, a high glycolytic flux may yield increased ATP in a shorter period than OXPHOS in the presence of an unlimited glucose supply (43). Previous studies $(20,23)$ demonstrated that stimuli factors such as ROS and 4-hydroxynonenal significantly augmented the glycolysis response to the decreased OCR in myocardial cells or bovine aortic endothelial cells. Unexpectedly, the present study demonstrated that AGEs negatively affected the glycolysis response. However, the latent molecular mechanisms underlying the observed AGEs-induced glycolytic dysfunction require further investigation. The present study hypothesized that AGEs may affect the activity or expression of crucial rate-limiting enzymes involved in glycolysis.

In conclusion, the results of the present study demonstrated that AGEs inhibited the viability and proliferation of intact HUVECs by negatively affecting mitochondrial aerobic respiration and glycolysis. These results may serve as a foundation for the further study of AGEs-induced endothelial dysfunction, which may provide a novel perspective for exploring therapeutic strategies for diabetic vascular complications.

\section{Acknowledgements}

The present study was supported by the National Natural Science Foundation of China (grant no. 81470417) and the Natural Science Foundation of Liaoning Province (grant no. 2013021090).

\section{References}

1. Yisahak SF, Beagley J, Hambleton IR and Narayan KM; IDF Diabetes Atlas: Diabetes in North America and the Caribbean: An update. Diabetes Res Clin Pract 103: 223-230, 2014.

2. Aso Y, Inukai T, Tayama K and Takemura Y: Serum concentrations of advanced glycation endproducts are associated with the development of atherosclerosis as well as diabetic microangiopathy in patients with type 2 diabetes. Acta Diabetol 37: 87-92, 2000.

3. Kiuchi K, Nejima J, Takano T, Ohta M and Hashimoto H: Increased serum concentrations of advanced glycation end products: A marker of coronary artery disease activity in type 2 diabetic patients. Heart 85: 87-91, 2001.

4. Genuth S, Sun W, Cleary P, Sell DR, Dahms W, Malone J, Sivitz W and Monnier VM; DCCT Skin Collagen Ancillary Study Group: Glycation and carboxymethyllysine levels in skin collagen predict the risk of future 10-year progression of diabetic retinopathy and nephropathy in the diabetes control and complications trial and epidemiology of diabetes interventions and complications participants with type 1 diabetes. Diabetes 54: 3103-3111, 2005.

5. Xu L, Zang P, Feng B and Qian Q: Atorvastatin inhibits the expression of RAGE induced by advanced glycation end products on aortas in healthy Sprague-Dawley rats. Diabetol Metab Syndr 6: 102, 2014.

6. Adamopoulos C, Piperi C, Gargalionis AN, Dalagiorgou G, Spilioti E, Korkolopoulou P, Diamanti-Kandarakis E and Papavassiliou AG: Advanced glycation end products upregulate lysyl oxidase and endothelin-1 in human aortic endothelial cells via parallel activation of ERK1/2-NF- $x$ B and JNK-AP-1 signaling pathways. Cell Mol Life Sci 73: 1685-1698, 2016. 
7. Goldin A, Beckman JA, Schmidt AM and Creager MA Advanced glycation end products: Sparking the development of diabetic vascular injury. Circulation 114: 597-605, 2006.

8. Kishikawa H, Mine S, Kawahara C, Tabata T, Hirose A, Okada Y and Tanaka Y: Glycated albumin and cross-linking of CD44 induce scavenger receptor expression and uptake of oxidized LDL in human monocytes. Biochem Biophys Res Commun 339: 846-851, 2006.

9. Nangaku M, Miyata T, Sada T, Mizuno M, Inagi R, Ueda Y, Ishikawa N, Yuzawa H, Koike H, van Ypersele de Strihou C and Kurokawa K: Anti-hypertensive agents inhibit in vivo the formation of advanced glycation end products and improve renal damage in a type 2 diabetic nephropathy rat model. J Am Soc Nephrol 14: 1212-1222, 2003.

10. Thomas MC, Baynes JW, Thorpe SR and Cooper ME: The role of AGEs and AGE inhibitors in diabetic cardiovascular disease. Curr Drug Targets 6: 453-474, 2005.

11. Machado AP, Pinto RS, Moysés ZP, Nakandakare ER, Quintão EC and Passarelli M: Aminoguanidine and metformin prevent the reduced rate of HDL-mediated cell cholesterol efflux induced by formation of advanced glycation end products. Int J Biochem Cell Biol 38: 392-403, 2006.

12. Goh SY and Cooper ME: Clinical review: The role of advanced glycation end products in progression and complications of diabetes. J Clin Endocrinol Metab 93: 1143-1152, 2008.

13. Steenvoorden MM, Huizinga TW, Verzijl N, Bank RA, Ronday HK, Luning HA, Lafeber FP, Toes RE and DeGroot J: Activation of receptor for advanced glycation end products in osteoarthritis leads to increased stimulation of chondrocytes and synoviocytes. Arthritis Rheum 54: 253-263, 2006.

14. Fuentes MK, Nigavekar SS, Arumugam T, Logsdon CD, Schmidt AM, Park JC and Huang EH: RAGE activation by S100P in colon cancer stimulates growth, migration, and cell signaling pathways. Dis Colon Rectum 50: 1230-1240, 2007.

15. Leclerc E, Fritz G, Weibel M, Heizmann CW and Galichet A: S100B and S100A6 differentially modulate cell survival by interacting with distinct RAGE (receptor for advanced glycation end products) immunoglobulin domains. J Biol Chem 282: 31317-31331, 2007.

16. Kim S and Kwon J: Actin cytoskeletal rearrangement and dysfunction due to activation of the receptor for advanced glycation end products is inhibited by thymosin beta 4 . J Physiol 593 1873-1886, 2015.

17. Del Turco S, Navarra T, Gastaldelli A and Basta G: Protective role of adiponectin on endothelial dysfunction induced by AGEs: A clinical and experimental approach. Microvasc Res 82: 73-76, 2011.

18. Lin J, Tang Y, Kang Q, Feng Y and Chen A: Curcumin inhibits gene expression of receptor for advanced glycation end-products (RAGE) in hepatic stellate cells in vitro by elevating PPAR activity and attenuating oxidative stress. Br J Pharmacol 166 : 2212-2227, 2012

19. Hill BG, Benavides GA, Lancaster JR Jr, Ballinger S, Dell'Italia L, Jianhua $Z$ and Darley-Usmar VM: Integration of cellular bioenergetics with mitochondrial quality control and autophagy. Biol Chem 393: 1485-1512, 2012.

20. Hill BG, Dranka BP, Zou L, Chatham JC and Darley-Usmar VM: Importance of the bioenergetic reserve capacity in response to cardiomyocyte stress induced by 4-hydroxynonenal. Biochem J 424: 99-107, 2009

21. Mitra K, Wunder C, Roysam B, Lin G and Lippincott-Schwartz J: A hyperfused mitochondrial state achieved at G1-S regulates cyclin E buildup and entry into S phase. Proc Natl Acad Sci USA 106: 11960-11965, 2009.

22. Kroemer G, Galluzzi L and Brenner C: Mitochondrial membrane permeabilization in cell death. Physiol Rev 87: 99-163, 2007.

23. Dranka BP, Hill BG and Darley-Usmar VM: Mitochondrial reserve capacity in endothelial cells: The impact of nitric oxide and reactive oxygen species. Free Radic Biol Med 48: 905-914, 2010.

24. Higdon AN, Benavides GA, Chacko BK, Ouyang X, Johnson MS, Landar A, Zhang J and Darley-Usmar VM: Hemin causes mitochondrial dysfunction in endothelial cells through promoting lipid peroxidation: The protective role of autophagy. Am J Physiol Heart Circ Physiol 302: H1394-H1409, 2012.
25. Ballinger SW, Patterson C, Knight-Lozano CA, Burow DL, Conklin CA, Hu Z, Reuf J, Horaist C, Lebovitz R, Hunter GC, et al: Mitochondrial integrity and function in atherogenesis. Circulation 106: 544-549, 2002

26. Lee J, Giordano S and Zhang J: Autophagy, mitochondria and oxidative stress: Cross-talk and redox signalling. Biochem J 441: 523-540, 2012.

27. Hori O, Ichinoda F, Tamatani T, Yamaguchi A, Sato N, Ozawa K, Kitao Y, Miyazaki M, Harding HP, Ron D, et al: Transmission of cell stress from endoplasmic reticulum to mitochondria: Enhanced expression of Lon protease. J Cell Biol 157: 1151-1160, 2002.

28. Kizhakekuttu TJ, Wang J, Dharmashankar K, Ying R, Gutterman DD, Vita JA and Widlansky ME: Adverse alterations in mitochondrial function contribute to type 2 diabetes mellitus-related endothelial dysfunction in humans. Arterioscler Thromb Vasc Biol 32: 2531-2539, 2012.

29. Hernandez-Mijares A, Rocha M, Rovira-Llopis S, Bañuls C, Bellod L, de Pablo C, Alvarez A, Roldan-Torres I, Sola-Izquierdo E and Victor VM: Human leukocyte/endothelial cell interactions and mitochondrial dysfunction in type 2 diabetic patients and their association with silent myocardial ischemia. Diabetes Care 36: 1695-1702, 2013.

30. Ferrick DA, Neilson A and Beeson C: Advances in measuring cellular bioenergetics using extracellular flux. Drug Discov Today 13: 268-274, 2008

31. Gottlieb E, Armour SM, Harris MH and Thompson CB: Mitochondrial membrane potential regulates matrix configuration and cytochrome c release during apoptosis. Cell Death Differ 10: 709-717, 2003.

32. Lei T, Guo N, Tan MH and Li YF: Effect of mouse oocyte vitrification on mitochondrial membrane potential and distribution. J Huazhong Univ Sci Technolog Med Sci 34: 99-102, 2014.

33. Luft VC, Duncan BB, Schmidt MI, Chambless LE, Pankow JS, Hoogeveen RC, Couper DJ and Heiss G: Carboxymethyl lysine, an advanced glycation end product, and incident diabetes: A case-cohort analysis of the ARIC Study. Diabet Med 33: 1392-1398, 2016

34. Sang HQ, Gu JF, Yuan JR, Zhang MH, Jia XB and Feng L: The protective effect of Smilax glabra extract on advanced glycation end products-induced endothelial dysfunction in HUVECs via RAGE-ERK1/2-NF- $\mathrm{B}$ pathway. J Ethnopharmacol 155: 785-795, 2014

35. Rempel LC, Finco AB, Maciel RA, Bosquetti B, Alvarenga LM, Souza WM, Pecoits-Filho R and Stinghen AE: Effect of PKC- $\beta$ signaling pathway on expression of MCP-1 and VCAM-1 in different cell models in response to advanced glycation end products (AGEs). Toxins (Basel) 7: 1722-1737, 2015.

36. Solé M, Miñano-Molina AJ and Unzeta M: Cross-talk between $\mathrm{A} \beta$ and endothelial SSAO/VAP-1 accelerates vascular damage and $\mathrm{A} \beta$ aggregation related to CAA-AD. Neurobiol Aging 36: 762-775, 2015.

37. Tang X, Luo YX, Chen HZ and Liu DP: Mitochondria, endothelial cell function, and vascular diseases. Front Physiol 5: 175, 2014.

38. Brand MD and Nicholls DG: Assessing mitochondrial dysfunction in cells. Biochem J 435: 297-312, 2011.

39. Shenouda SM, Widlansky ME, Chen K, Xu G, Holbrook M, Tabit CE, Hamburg NM, Frame AA, Caiano TL, Kluge MA, et al: Altered mitochondrial dynamics contributes to endothelial dysfunction in diabetes mellitus. Circulation 124: 444-453, 2011.

40. Ong SB, Subrayan S, Lim SY, Yellon DM, Davidson SM and Hausenloy DJ: Inhibiting mitochondrial fission protects the heart against ischemia/reperfusion injury. Circulation 121: 2012-2022, 2010.

41. Schleicher E and Friess U: Oxidative stress, AGE, and atherosclerosis. Kidney Int Supp S17-S26, 2007.

42. Groschner LN, Waldeck-Weiermair M, Malli R and Graier WF: Endothelial mitochondria-less respiration, more integration. Pflugers Arch 464: 63-76, 2012.

43. Eelen G, de Zeeuw P, Simons M and Carmeliet P: Endothelial cell metabolism in normal and diseased vasculature. Circ Res 116: 1231-1244, 2015. 